\title{
Research and Improvement of LTE-A Uplink Frequency Offset Estimation
}

\author{
Zhaohua ZENG ${ }^{1, a}$, Xueping LIU $^{2, b}$ \\ ${ }^{1}$ College of Communication and Information Engineering, Xi'an University of Science and \\ Technology , Xi'an, 710054, China \\ ${ }^{2}$ College of Communication and Information Engineering, Xi'an University of Science and \\ Technology , Xi'an, 710054, China

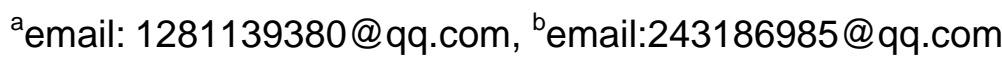

Keywords: LTE-A ; frequency offset estimation; fixed frequency offset; Doppler frequency offset

\begin{abstract}
In LTE-A system, there are generally two types of frequency offset: fixed frequency offset and Doppler frequency offset. In the paper, firstly, the estimation of fixed frequency offset and Doppler frequency offset is introduced, then several improved estimation schemes is proposed. Secondly, with the presence of two types of frequency offset in the system, a joint estimation and compensation algorithm of fixed frequency offset and Doppler frequency offset is proposed. Simulation results show that the proposed algorithms can meet the performance requirements for frequency synchronization of LTE-A system and reduce system block error ratio effectively.
\end{abstract}

\section{Introduction}

When signal is transmitted on wireless channel in LTE-A system, because the oscillation frequency exist small gap between transmitter and receiver or UE generated by the movement of the Doppler frequency shift will lead to sub carrier not orthogonal, so that system performance deterioration. Therefore, in order to improve the performance of the system, it is necessary to estimate and compensate the frequency offset of the uplink transmission system.

Literature[1][2][3] aiming at the characteristics of OFDM/OFDMA/SC-FDMA transmission system in frequency offset estimation and tracking, some algorithms are proposed to estimate and track the frequency shift of Doppler. These methods can be roughly divided into the following two categories: the first kind is based on pilot assisted estimation algorithm for systems, the core idea of this algorithm is the information symbols at the transmitter inserting a plurality of pilot data, after wireless transmission at the receiving end of the FFT transform, using the pilot position of the data information to estimate the system Doppler frequency offset[4]. The second estimation method for the blind estimation method, without the use of auxiliary data, but to use the symbol structure of special treatment, such as cyclic prefix (CP), decision data, processing of data on the number of subcarrier symbols estimated Doppler frequency offset value[5]. In this type of method, some research is based on the frequency offset estimation of CP and the method of Judging according to the direction of the data. To compare the two methods shows that based on pilot assisted occupy a dominant position in the complexity, and it is more adopted real - time high - speed system, but the efficiency of information transmission is reduced due to the non - carrying of useful information in the pilot position. Blind estimation algorithm has higher complexity and lower SNR performance, but it does not need to insert the pilot symbols to improve the transmission efficiency. Therefore, the frequency offset estimation in high-speed real-time system is the focus of the research at home and abroad.

\section{Estimation of fixed frequency offset and Doppler offset frequency combination}

The frequency offset in the system can be estimated based on two aspects: fist used received signal in time domain to calculate, superposition of multi user data,and it is not easy to calculate; 
second, used the frequency domain received pilot to calculate, To calculate the frequency offset in a certain range according to the between pilot the phase difference and time interval between pilot bits.

When uplink PUSCH channel without hopping and frequency offset estimation can be achieved through two slots in a sub frame pilot channel estimation information acquisition. According to the two pilot bits corresponding to the same sub carrier frequency channel estimation information angle difference occurred in the time frequency offset phase difference, and get the value of frequency deviation. Therefore, the frequency offset estimate is obtained through the direct pilot channel estimation in frequency domain.

(1) Fixed frequency offset estimation

The scheme is as follows:

In each sub carrier wave band, the frequency offset is estimated, and then the frequency offset of the frame is obtained according to the power weighted combination.

$$
\begin{aligned}
& \left|H_{i, k a}\right|^{2}=\sum_{j=1}^{M / Q} H_{i, j, k a} \times H_{i, j, k a}^{*} \\
& R_{i, k a}=\frac{\left|H_{n, k a}^{i}\right|^{2}+\left|H_{n+m, k a}^{i}\right|^{2}}{\sum_{i}^{Q}\left(\left|H_{n, k a}^{i}\right|^{2}+\left|H_{n+m, k a}^{i}\right|^{2}\right)} \\
& \Delta \Delta f_{i, k a}=\frac{\angle\left(\bar{H}_{n+m, k a}^{i}\right)-\angle\left(\bar{H}_{n, k a}^{i}\right)-2 \pi \cdot m \cdot t \cdot \overline{\Delta f}_{n_{-} \text {subframe- } 1, k a}}{2 \pi \cdot m \cdot t}+l \times 2000 \\
& =\frac{\angle\left(\bar{H}_{n+m, k a}^{i}\right)-\angle\left(\bar{H}_{n, k a}^{i}\right)}{2 \pi \times 0.5 \times 10^{-3}}-\overline{\Delta f}_{n_{-} \text {subframe- } 1, k a}+l \times 2000 \\
& =\frac{\angle\left(\bar{H}_{n+m, k a}^{i} / \bar{H}_{n, k a}^{i}\right)}{2 \pi \times 0.5 \times 10^{-3}}-\overline{\Delta f}_{n_{-} \text {subframe-1,kat }}+l \times 2000
\end{aligned}
$$

$n_{-}$subframe is frame number.

$$
\Delta \Delta f_{k a}=\sum_{i=1}^{Q} \Delta \Delta f_{i, k a} \times R_{i, k a}
$$

(2) Estimation of fixed frequency offset and Doppler offset frequency combination

The frequency offset estimation algorithm based on pilot frequency is as follows of :

1) To get target user's frequency domain data:

$d(n)$ is receiving data in time domain , By FFT transform to the frequency domain is $D(k)$, according to the target user in the frequency domain of the physical location of the target user is located in the sub carrier of the pilot symbols of the 2 slots, according to the target user in the frequency domain of the physical location obtained the pilot symbols of the 2 slots in the sub carrier of the target user, the pilot symbols of the 2 slots is $Y_{1}(k), Y_{2}(k), 1 \leq \mathrm{k} \leq \mathrm{M}$ M as the target users of the number of sub carriers.

2) To calculate the estimate value of the pilot

Suppose $Y_{1}(k)$ is receive the sequence of the frequency domain of pilot $1, Y_{2}(k)$ is receive the sequence of the frequency domain of pilot 2, the local frequency pilot code is $X(k)$, then the corresponding channel estimation can be expressed as:

$$
\begin{aligned}
& H_{1}(k)=\frac{Y_{1}(k)}{X(k)}=a_{k} e^{j \phi_{1}}, 1 \leq \mathrm{k} \leq \mathrm{M} \\
& H_{2}(k)=\frac{Y_{2}(k)}{X(k)}=b_{k} e^{j \phi_{2}}, 1 \leq \mathrm{k} \leq \mathrm{M}
\end{aligned}
$$

where $\phi_{1}$ and $\phi_{2}$ contain the channel phase property and frequency offset phase information at $\mathrm{t}_{1}$ and $\mathrm{t}_{2} ; 1 \leq k \leq M, \mathrm{M}$ is the number of sub-carriers occupied by the target user.

3) To get the sub carrier frequency offset 
Assuming that the channel is flat-fading in time domain, namely it does not change or change a bit during the interval $\Delta t=t_{2}-t_{1}$ between pilot1 and pilot2. Thus frequency offset can be calculated based on the difference of phase between phase $\phi_{1}$ and phase $\phi_{2}$, which can be expressed as

$$
\Delta f(k)=\frac{\phi_{2}(k)-\phi_{1}(k)}{2 \pi \Delta t}=\frac{\angle H_{2}(k)-\angle H_{1}(k)}{2 \pi \times \frac{1 \times 10^{-3}}{14}\left(n_{2}-n_{1}\right)} 1 \leq k \leq M
$$

Where $n_{2}-n_{1}$ is the two pilot symbols between the number of OFDM, $\square$ a sub frame length is $1 \mathrm{~ms}$, and a total symbols are 14 OFDM symbols, so the time between adjacent two symbols is $\frac{1 \times 10^{-3}}{14}$ (s: seconds).

It can be seen from the above formula, the maximum value of frequency offset estimation $\Delta f(k)$ is related of $\Delta t$, the $\Delta t$ more smaller, the $\Delta f(k)$ greater the larger.

In the non frequency hopping mode of PUSCH, the DMRS direct distance is 7 OFDM symbols on two slots, then there is:

$$
\Delta f(k)=\frac{\angle H_{2}(k)-\angle H_{1}(k)}{2 \pi \times \frac{1 \times 10^{-3}}{14} \times 7}=\frac{\angle H_{2}(k)-\angle H_{1}(k)}{2 \pi \times 5 \times 10^{-4}}
$$

Because the range of phase is from $-\pi$ to $\pi\left(-\pi \leq \phi_{2}(\mathrm{k})-\phi_{1}(\mathrm{k}) \leq \pi\right)$, so the range of frequency estimation is from $-1000 \mathrm{~Hz}$ to $1000 \mathrm{~Hz}(-1000 \mathrm{~Hz} \leq \Delta f(k) \leq 1000 \mathrm{~Hz})$.

4) To calculate frequency offset estimation of target user

The calculation of the frequency offset on the multi carrier can be divided into 2 ways:

A. equal weight to the average

$$
\Delta f=\frac{1}{M} \sum_{k=1}^{M} \Delta f(k)
$$

B. According to the power of $\mathrm{H}$ as the weight of the average

Each adjacent sub carrier is divided into a segment, which is divided into Q segments, each segment of the power sum:

$$
\begin{gathered}
\left|H_{i}\right|^{2}=\sum_{j=1}^{M / Q} H_{i, j} \times H_{i, j}^{*} \\
R_{i}=\frac{\left|H_{i}\right|^{2}}{\sum_{i}^{Q}\left|H_{i}\right|^{2}} \\
\Delta f(i)=\frac{\phi_{2}(i)-\phi_{1}(i)}{2 \pi \Delta t} \\
\Delta f=\sum_{i=1}^{Q} \Delta f(i) \times R_{i}
\end{gathered}
$$

5) To smooth between sub frames

$$
\overline{\Delta f_{n_{-} \text {frame }}}=(1-p) \cdot \overline{\Delta f_{n_{-} \text {frame- } 1}}+p \cdot \Delta f
$$

Where $\mathrm{P}$ is the filter factor, $0 \leq p \leq 1$.

6) To process receiving frequency offset of multi-antenna

When receiving antenna between the vibration, and the distance between the small enough, you can think each receive antenna frequency offset approximately equal, different antenna frequency offset estimation value and a correction value are the same, each antenna unified frequency offset estimation and correction can effectively reduce the computational complexity.

When the receiving antenna between crystal different, large space, each antenna frequency partial is different, so different receiving antenna frequency offset estimation value, a correction 
value needs to be processed separately, frequency offset estimation and correction calculation is times of a Ka of the former kind, Ka is the number of the antennas.

7) To smooth of frequency domain in channel estimation

$H_{1}(k)$ and $H_{2}(k)$ are smoothed of frequency domain in channel estimation, each adjacent sub carrier is divided into a segment, which is divided into $Q$ segments, the average value of each segment:

$$
\begin{aligned}
& H_{1, i}=\sum_{j=1}^{M / Q} H_{1, i, j}, 1 \leq i \leq Q, 1 \leq j \leq M / Q \\
& H_{2, i}=\sum_{j=1}^{M / Q} H_{2, i, j}, 1 \leq i \leq Q, 1 \leq j \leq M / Q
\end{aligned}
$$

\section{Improved Method}

(1) Method1

In a multi antenna system, the frequency offset of each antenna is different, suppose $H(1, j), H(2, j)$ are pilot channel estimate value in different times on the antenna j, calculated frequency offset value use $H(1, j), H(2, j)$ :

$$
\Delta f(i, j)=\operatorname{angle}(H(2, j) \times \operatorname{conj}(H(1, j))) \times \frac{1}{2 \pi \times 0.5 \times 10^{3}}
$$

And use $H(2, j) \quad \Delta f(i, j)$ compensation obtain the new formula is $\tilde{H}(2, j)$ :

$$
\tilde{H}(2, j)=H(2, j) \times e^{-2 \pi \times 0.5 \times 10^{3} \times \Delta f(i, j)}
$$

Calculate data bit channel estimation, which using of $H(1, j), \tilde{H}(2, j)$ pilot, Finally, the frequency offset of the whole antenna is calculated by the power weighted:

$$
\Delta f(i)=\operatorname{angle}\left(\sum_{j=\text { ant }} \tilde{H}(2, j) \times \operatorname{conj}(H(1, j))\right) \times \frac{1}{2 \pi \times 0.5 \times 10^{3}}
$$

Finally, the use of the symbol of $\Delta f(i)$ in the frequency offset compensation .

(2) Method 3

At first, the frequency offset of $\mathrm{J}$ antenna is calculated at two different times:

$$
\Delta f(i, j)=\operatorname{angle}(H(2, j) \times \operatorname{conj}(H(1, j))) \times \frac{1}{2 \pi \times 0.5 \times 10^{3}}
$$

Then making smooth between the subframe, because the filter factor can effectively suppress the frequency offset estimation is not accurate;

$$
\Delta f(i, j)=\Delta f(i-1, j)+\alpha \times(\Delta f(i, j)-\Delta f(i-1, j))
$$

The value is then used to form a new $\tilde{H}(2, j)$ :

$$
\tilde{H}(2, j)=H(2, j) \times e^{-2 \pi \times 0.5 \times 10^{3} \times \Delta f(i, j)}
$$

Finally, the channel estimation is calculated using the data bit $H(1, j), \tilde{H}(2, j)$;

After equalization, the use of the making symbol offset compensation use of $\Delta f(i)$;

$$
\Delta f(i)=\operatorname{angle}\left(\sum_{j=a n t} \tilde{H}(2, j) \times \operatorname{conj}(H(1, j))\right) \times \frac{1}{2 \pi \times 0.5 \times 10^{3}}
$$

(3) Method 4

Firstly, all antennas in the time i frequency offset value is calculated by the weighted power; and smoothed frequency offset value lacy pilot phase, and compensate for intersymbol frequency offset;

$$
\Delta f(i)=\text { angle }\left(\sum_{j=a n t} H(2, j) \times \operatorname{conj}(H(1, j))\right) \times \frac{1}{2 \pi \times 0.5 \times 10^{3}}
$$

Then making smooth between the sub frame, 


$$
\Delta f(i)=\Delta f(i-1)+\alpha \times(\Delta f(i)-\Delta f(i-1))
$$

The value is then used to form a new $\tilde{H}(2, j)$ :

$$
\tilde{H}(2, j)=H(2, j) \times e^{-2 \pi \times 0.5 \times 10^{3} \times \Delta f(i)}
$$

Finally, the channel estimation is calculated using the data bit $H(1, j), \tilde{H}(2, j)$; After equalization, the use of the making symbol offset compensation use of $\Delta f(i)$;

\section{Simulation Results}

This simulation is platform in the LTE simulation, Parameter is set to: bandwidth is $20 \mathrm{M}$, the number of subcarriers of each OFDM symbol $\mathrm{N}=256$, uplink and downlink configuration is uplink, the number of $\mathrm{RB}$ is 1 , 100 . Number of antenna is $1 \mathrm{~T} 2 \mathrm{R}$; channel model are EVA5Hz and EVA70Hz, modulation is QPSK.

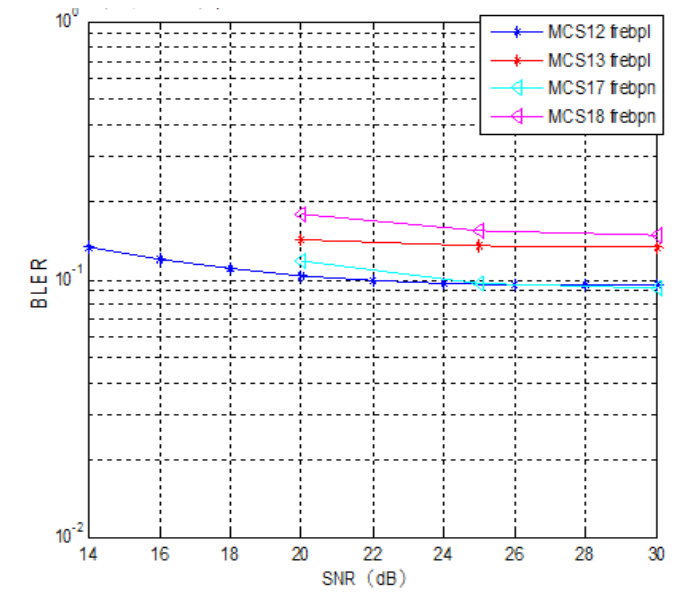

Fig.1 Performance comparison of different MCS

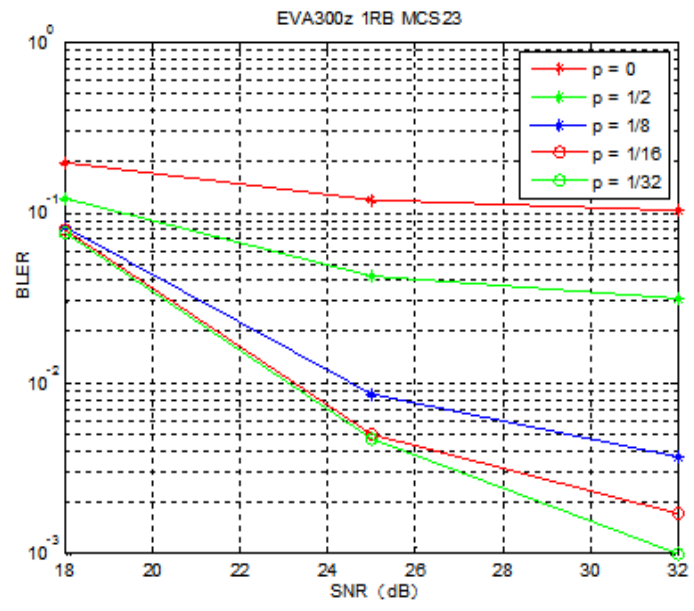

Fig.2 Performance comparison of different smoothing factors The performance comparison of different thing is illustrated in Fig.1 and Fig.2. We can see that from it. When the interference is presented, frequency offset have great impact on the size of RB's IRC; when fixed frequency offset unchanged, the smooth between TTI can bring gains, especially for existing proposed 1; from the simulation, the smoothing factor 1/8 convergence has been able to guarantee. For fixed frequency offset is exchanged, such as high-speed railway scene, the need for a tradeoff between speed and convergence of tracking, based on different scenarios simulation, the use of smoothing factor $1 / 2$.

\section{Conclusions}

In this paper, the effects of frequency offset on the system are studied. Firstly, the estimation of the combination of natural frequency offset and Doppler frequency offset is introduced, then, several improvements scheme of frequency offset are proposed, and the simulation analysis is presented. Simulation results show that the proposed algorithm can meet the performance requirements for frequency synchronization of LTE system and reduce system block error radio (BLER) and calculation.

\section{Acknowledgment}

This work was supported in part by the Science and Technology Plan of Shaanxi Province, China (Program No. 2013K07-35;2014K06-37), the Science and Technology Plan Industrial Application Technology Research and Development Project of Xi'an (Program No.CXY1440(4)), the Xi'an City Beilin District Application Technology Research and Development Project (Program No.GX1310; GX1311;GX1417). 


\section{References}

[1] Guo Pan, Li Zheng, "Effect of carrier frequency offset on OFDM and simulation analysis," Computer Simulation, 2007, vol.08, pp.283-285+294.

[2] H. Cheon. Frequency offset estimation for high speed users in E-UTRA uplink. In Proc. of IEEE International Symposium on Personal, Indoor and Mobile Radio Communic ations. September 2007, Athens, Greece, pp. 1-5.

[3] Van de Beek J J, Sandell M. ML estimation of time and frequency offset in OFDM systems. IEEE Trans. On Signal Processing. July 1997,45 (7), pp. 1800-1850.

[4] Ai B, Ge J H, Wang Y. Decimal Frequency Offset Estimation in COFDM Wireless Communications. IEEE Transactions on Broadcasting. June 2004, 50 (2), pp.154-158.

[5] Ai Bo, Wang Jintao, Zhong Zhangdui, "Synchronization technology of broadband wireless communication OFDM,” Posts \& Telecom, 2011, pp.4+88, in press.

[6]Yue Li, "Studies on phase noise mitigation of OFDM systems," Harbin Engineering University, 2007.

[7] Fatang Chen, Cui Wan, "Research and simulation for frequency offset estimation algorithm in TD-LTE system,” Computer Science and Electronic Technology International Society, 2014, vol.513-517, pp.1063-1066. 